\title{
First Spatial Alignment of the LHCb VELO and Analysis of Beam Absorber Collision Data
}

S. Borghi ${ }^{* a}$, M. Gersabeck ${ }^{\mathrm{a}}$, C. Parkes ${ }^{\mathrm{a}}$, T. Szumlak ${ }^{\mathrm{a}}$, A. Affolder ${ }^{\mathrm{b}}$, K. Akiba ${ }^{\mathrm{c}}$, J. Anderson ${ }^{\mathrm{h}}$, M. Artuso ${ }^{\mathrm{i}}$,

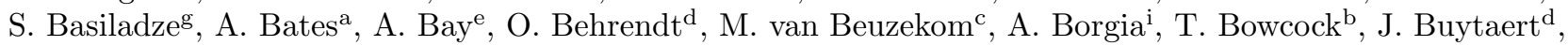

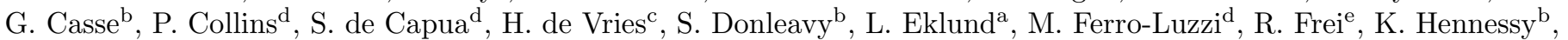
T. Huse ${ }^{\text {b }}$, D. Hutchcroft ${ }^{\mathrm{b}}$, E. Jans ${ }^{\mathrm{c}}$, M. John ${ }^{\mathrm{f}}$, T. Ketel ${ }^{\mathrm{c}}$, G. Lefeuvre ${ }^{\mathrm{i}}$, A. Leflat ${ }^{\mathrm{g}}$, F. Marinho ${ }^{\mathrm{a}}$, R. McNulty ${ }^{\mathrm{h}}$, R. Mountain ${ }^{\mathrm{i}}$, I. Mous ${ }^{\mathrm{c}}$, J. Mylroie-Smith ${ }^{\mathrm{b}}$, A. Noor ${ }^{\mathrm{b}}$, A. Papadelis ${ }^{\mathrm{c}}$, G. Patel ${ }^{\mathrm{b}}$, B. Rakotomiaramanana ${ }^{\mathrm{a}}$, K. Rinnert ${ }^{\mathrm{b}}$, E. Rodrigues ${ }^{\mathrm{a}}$, T. Shears ${ }^{\mathrm{b}}$, N.A. Smith ${ }^{\mathrm{b}}$, M. Tobin ${ }^{\mathrm{b}}$, S. Traynor ${ }^{\mathrm{h}}$, A. Van Lysebetten ${ }^{\mathrm{c}}$, B. Verlaat ${ }^{\mathrm{c}}$, J. Wang $^{\mathrm{i}}$

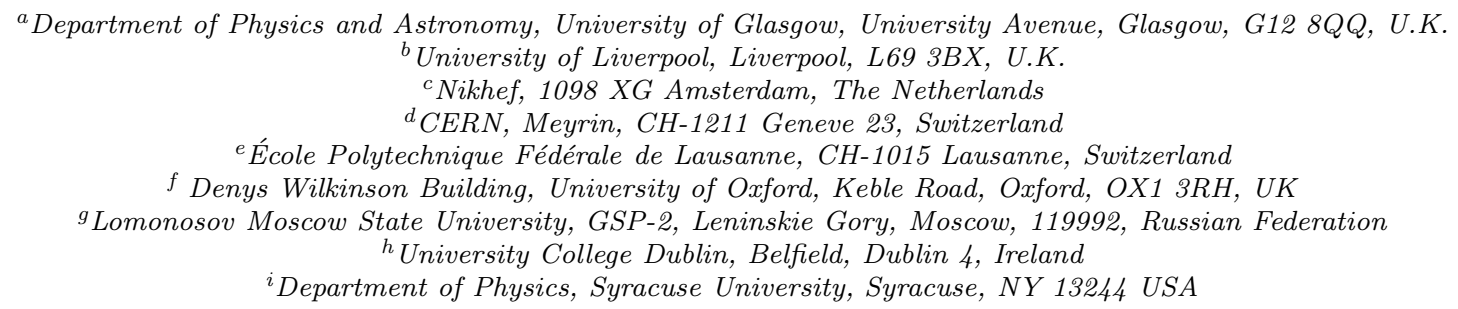

\begin{abstract}
A first alignment of the LHCb Vertex Locator has been obtained from beam induced tracks at the LHC. $450 \mathrm{GeV} / \mathrm{c}$ protons were collided on a beam absorber during the LHC synchronisation tests of the anti-clockwise beam in August and September 2008. The resulting particle tracks have been reconstructed by the Vertex Locator. This was the first full reconstruction of tracks induced by the LHC beam. The quality of the data obtained is discussed. A total of 2200 tracks were reconstructed from the full data sample, and a first spatial alignment was obtained. The detector is aligned to an accuracy of $5 \mu \mathrm{m}$ in the sensor plane. The results confirm that all detector modules have not been displaced from their surveyed positions by more than $10 \mu \mathrm{m}$.
\end{abstract}

Key words: Silicon, Microstrip, Vertex Detector, Alignment, LHCb, LHC

\section{Introduction}

The Large Hadron Collider (LHC) at CERN will collide bunches of up to $10^{11}$ protons (and $7 \cdot 10^{7}$ heavy ions) at a centre of mass energy up to $14 \mathrm{TeV}(5.5 \mathrm{TeV})$ and a design luminosity up to $10^{34} \mathrm{~cm}^{-2} \mathrm{~s}^{-1}\left(10^{27} \mathrm{~cm}^{-2} \mathrm{~s}^{-1}\right)$. The four large detectors, (ALICE[1], ATLAS[2], CMS[3], $\mathrm{LHCb}[4])$ are currently in their commissioning phase. Cosmic ray tracks have been observed in multiple sub-systems of the LHC experiments from 2006 onwards, and regular data taking of cosmic rays in several sub-systems of the experiments took place during 2008 as the final construction of the experiments was completed. The data have been used for the sub-detector calibration and alignment $[5,6,7]$. However, as explained below, the layout of the vertex detector is not conducive to being commissioned in situ with cosmic rays. This paper reports on data quality checks and spatial alignment of the VELO based on data from LHC injection tests.

\footnotetext{
${ }^{*}$ Corresponding author. Tel.: +41-22-76-72670.

Email address: Silvia.Borghi@cern.ch (S. Borghi)
}

The alignment of each detector is crucial in reaching the required tracking performance in all LHC experiments. Typically, the initial alignment is evaluated by survey measurement at different stages of the assembly, and the final alignment procedure is based on track reconstruction [8]. The first evaluation of the track based alignment of the $\mathrm{LHCb}$ vertex detector has been performed with data recorded during the beam commissioning.

The LHCb experiment is dedicated to heavy flavour physics and it has been primarily designed to study $\mathrm{CP}$ violation and other rare phenomena in $b$-hadron decays. For a nominal luminosity of $2 \cdot 10^{32} \mathrm{~cm}^{-2} \mathrm{~s}^{-1}$ and for collisions at a centre-of-mass energy of $14 \mathrm{TeV}$, the expected production yield of $b \bar{b}$ quark pairs is about $10^{12}$ per year. $\mathrm{LHCb}$ (shown in fig. 1) is a forward-angle spectrometer with an angular coverage of 15-300 mrad. The track reconstruction [10] near the interaction point is performed by the silicon microstrip VErtex LOcator (VELO) [9] which is positioned around the primary impact point. The VELO provides precise tracking coordinates to reconstruct the position of the primary vertex and identify the displaced 


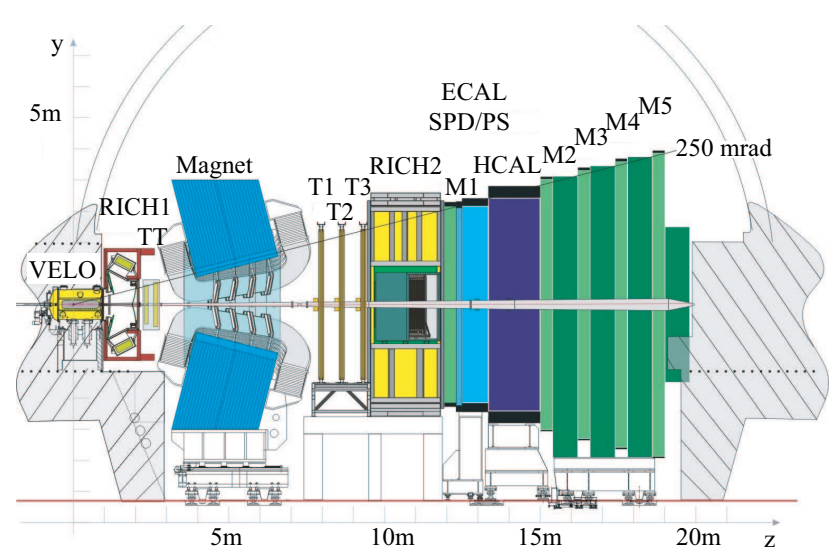

Figure 1: The LHCb detector setup with the different sub-detectors in the longitudinal plane. The Vertex Locator is shown on the lefthand side of the diagram and the other sub-detectors of the spectrometer are indicated.

vertices, which are a distinctive feature of $b$-hadron decays. The LHCb trigger system uses the collected data to enrich the $b$-content of the selected events in the high level trigger. The tracking system consists also of a silicon microstrip detector, named the Tracker Turicensis (TT) in front of the spectrometer magnet, and three tracking stations behind the magnet. The tracking system is expected to give a precision on reconstructed $\mathrm{B}$ hadron masses of $15-20 \mathrm{MeV} / \mathrm{c}^{2}$ and a proper time resolution of about 40 fs. The Cherenkov detector system (RICH1 and RICH2) provides excellent $\pi / K$ separation in the momentum range between 2 and $100 \mathrm{GeV} / \mathrm{c}$ : the average efficiency for kaon identification is $\epsilon(\mathrm{K} \rightarrow \mathrm{K}) \sim 95 \%$ with a corresponding average pion misidentification rate $\epsilon(\pi \rightarrow \mathrm{K}) \sim 5 \%$. The calorimeter system (PS, ECAL, HCAL) and the muon chambers provide electron and muon identification respectively. The particle identification is essential for signal selection and background rejection in many exclusive B channels as well as for flavour tagging.

Synchronisation tests of the LHC beam were performed in August and September 2008. During the initial phase of each test, a beam containing single bunches of protons was collided with a beam absorber in the transfer line between the CERN Super Proton Synchrotron (SPS) and the LHC. LHCb detectors measured some of the particles produced by the proton interactions in the absorber and by their re-interaction. This test provided the first reconstructed tracks after installation. The data sample has been extensively used for commissioning the detector and the first alignment of the VELO has been obtained with this sample.

The VELO has fully reconstructed about 2200 tracks traversing the detector. This sample provided the first opportunity to optimise the ADC sampling time of the sensors with respect to the SPS/LHC clock. The data reconstruction parameters had also not been optimised before these runs. In this paper the quality of the data sample

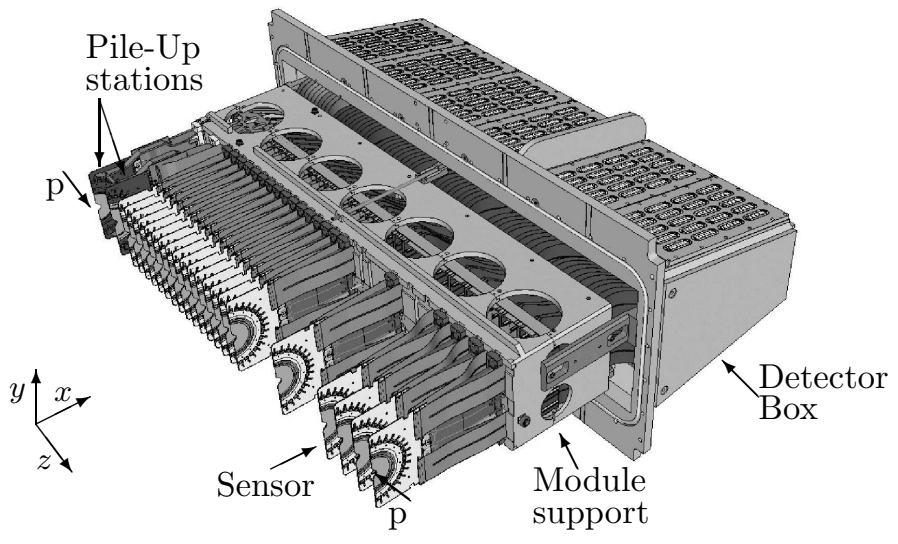

Figure 2: Overview of the VELO left half. The sensors, module supports and detector box are indicated.

obtained is discussed, and the improvements obtained by optimising the reconstruction parameters are presented. The results of aligning this detector are then provided.

This paper is structured as follows: a brief description of the VELO and of the commissioning is given in Section 2; the synchronisation test is described in Section 3 and the VELO data taking configuration in Section 4; the calibration of the VELO timing is then discussed in Section 5; the track reconstruction is presented in Section 6; Section 7 illustrates the results obtained by offline reprocessing of the data with the correct parameters for the data acquisition boards; Section 8 describes the module alignment results; the measured spatial resolution of the VELO is shown in Section 9; Section 10 summarises the main conclusions.

\section{VELO Description and Commissioning}

The VELO consists of two detector halves. The socalled "A-side" and "C-side" correspond to the positive and negative $x$ halves $^{1}$, respectively (shown in fig. 2). Radial and azimuthal hit coordinates are provided by 21 modules, each contains $R$ and $\Phi$ semi-circular $\mathrm{n}^{+}$-on-n silicon sensors perpendicular to the beam-axis. In addition, each half contains two Pile-Up veto stations used by the trigger system to reject events with more than one interaction. The detectors are operated in vacuum. The LHC beam vacuum is separated from the detector vacuum by $300 \mu \mathrm{m}$ thick aluminium foils mounted on each side for $\mathrm{RF}$ shielding and protection of the primary LHC vacuum from detector outgassing.

The large flux of secondary particles produced in the collisions constitutes an extreme radiation environment

\footnotetext{
${ }^{1}$ The coordinate system is shown in fig. 2 . The origin is the nominal interaction point, the $x$-axis is horizontal, and points from the interaction point towards the outside of the LHC ring. The $y$-axis is perpendicular to the $x$-axis and to the beam line and points upwards. The $z$-axis point from the interaction point towards the LHCb detector and is aligned with the beam direction, to create a right-handed Cartesian coordinate system.
} 
with highly non uniform particle fluences across the VELO sensors: the maximum fluence at nominal luminosity has been estimated to $1.4 \cdot 10^{14} \mathrm{MeV}$ neutron equivalents $/ \mathrm{cm}^{2}$ per year. The VELO cooling system is designed to absorb the heat generated in the sensor electronics and to minimize radiation induced effects in the silicon sensors by maintaining them at a temperature below $-5^{\circ} \mathrm{C}$.

The VELO halves are movable to the aperture required for the operation of the LHC machine. During collisions at $7 \mathrm{TeV}$, the halves are closed and the sensors overlap by a few millimeters to form complete circles with a radius of $7 \mathrm{~mm}$. However, the required LHC aperture increases during injection and energy ramping, and the detector halves have to be retracted by $30 \mathrm{~mm}$ from the beam axis. This is achieved with a precise motion system capable of positioning the VELO in the $x$ - and $y$ - directions with an accuracy of $10 \mu \mathrm{m}$. The same system can correct for variations of the beam position from fill to fill. The online imaging of the beams is provided by fast tracking and vertexing algorithms, also used to determine the motion steps and final detector position around the interaction point.

The VELO operation is assured by a control and data acquisition (DAQ) system. The main goals of this system are to power, cool and configure the front-end electronics; to provide the sensor bias voltage; to read out the signals; and to move and evacuate the detector volume to the required pressure. The analogue multiplexed signals of the 84 sensors are transmitted over $60 \mathrm{~m}$ cables to the TELL1 [11] readout boards responsible for the digitisation of the analogue data. The data are processed by FPGAs which subtract the pedestals, correct for common mode noise and cross-talk and finally perform the strip clusterisation [12]. The data are then sent to the filter farm of the $\mathrm{LHCb}$ experiment via a Gigabit Ethernet connection.

Tests of the DAQ software, detector control, tracking, vertexing and online monitoring were performed in 2006 [13], when a VELO half was partially assembled for a test beam. On that occasion, more than 50 million events were collected in various configurations. A signal-to-noise ratio of 20-24 (24-29) was measured for $R$-sensors ( $\Phi$-sensors). The installation of major components of the VELO system was completed in October 2007, and the full system has been operated since June 2008. The detector was operated under vacuum (with a pressure of $9 \cdot 10^{-7} \mathrm{mbar}$ in the detector volume achieved after 18 weeks of pumping). The cooling temperature was regulated at $-10^{\circ} \mathrm{C}$ and an average sensor temperature of $5^{\circ} \mathrm{C}$. More than 100 million events have been taken in random trigger and test pulse runs with a maximum trigger rate of $100 \mathrm{kHz}$.

\section{Beam Synchronisation Test}

In the synchronisation test of the LHC beam a single bunch of protons was sent along a transfer line from the SPS to the LHC. Before injecting the beam into the LHC, the bunch was collided on a beam absorber (TED: Transfer line External beam Dump [14]) to allow the injection

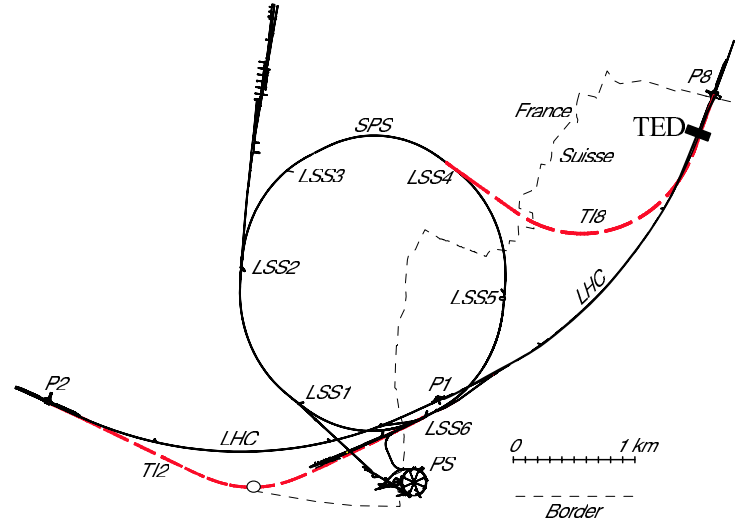

Figure 3: The layout of the transfer lines (dashed thick line) from the CERN Super Proton Synchrotron (SPS) to the Large Hadron Collider (LHC). The LHCb experiment is located at P8. Reproduced from [17].

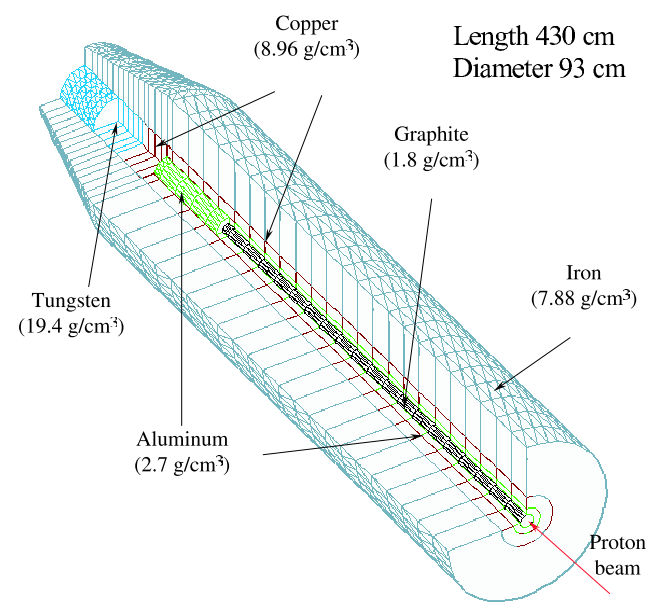

Figure 4: A schematic view of the TED, reproduced from [16].

line to be set-up. The TEDs are located in the two transfer lines from the SPS to the LHC (see fig. 3). They are $4.3 \mathrm{~m}$ long, consist of a stack of absorbers and include a graphite core (as shown in fig. 4). The TEDs are capable of absorbing the injected beam and protecting the LHC tunnel [15]. A flux of secondary particles (mostly muons) [16] ends up in the nearby LHC experimental areas (LHCb for injection line TI8 and ALICE for injection line TI2). Muons and some secondary particles coming from proton interaction in the TED are directed towards the experimental areas.

The TED in the injection line of beam 2 (anti-clockwise beam) is at about $340 \mathrm{~m}$ from the LHCb cavern with a horizontal deflection of about $12 \mathrm{mrad}$. The beam is injected vertically in the LHC by a deflecting kicker with a nominal total kick strength of $0.85 \mathrm{mrad}$ [18]. The particles generated in the collisions between the proton beam and the TED absorber pass through the LHC tunnel or scatter in the walls, with their products reaching the $\mathrm{LHCb}$ experiment. These particles traverse the $\mathrm{LHCb}$ detector in the reverse direction, i.e. entering first the muon stations and then emerging through the VELO detector. 
During the synchronisation test in August and September 2008 the LHCb magnet was switched off. In this test, a single bunch beam of about $5 \cdot 10^{9}$ protons of momentum $450 \mathrm{GeV} / \mathrm{c}$ was directed on the TED every 48 seconds for several hours.

As the TED was designed to be capable of absorbing a beam of $10^{13} 450 \mathrm{GeV} / \mathrm{c}$ protons every $17 \mathrm{~s}$, there was no risk in operating the sensitive VELO modules during these collisions. After the TED collisions, the beam was injected into the LHC and collided with a mobile beam stopper, and later injected through LHCb. The VELO was turned off during these later phases in order to prevent any damage to the detector.

\section{Detector and data taking configuration of the VELO}

A special detector configuration and software for the synchronisation tests were prepared: the triggering and timing were set up; the data acquisition was tested in the required configuration; and special on-line and off-line monitoring system was commissioned.

In order to observe tracks in the VELO, the timing was set up with respect to a trigger signal from the scintillator pad detector of the LHCb calorimeter system [10]. The front-end chip [19] of the VELO has a $40 \mathrm{MHz}$ sampling frequency (the LHC beam crossing frequency) and it is usually configured so that the signal pulse is primarily contained within one $25 \mathrm{~ns}$ time interval. However, for one data taking period (labelled A Sample in table 1) the front-end chip was set up to have a $25 \%$ longer pulse shape which increased the signal to noise of the detectors and thus maximised the probability of cleanly observing particles. As the sampling time with respect to the trigger could only be tested with the beam, the VELO was set up to read out several consecutive time samples spaced by 25 ns.

The VELO TELL1 boards [11] perform the raw signal processing up to strip clusterisation [12]. During these tests, only a basic configuration of the TELL1 algorithms [20] was set. A constant pedestal correction (set to 512 ADC counts) was used for all the channels, despite significant pedestal variations. A linear common mode correction was applied which helped reduce the pedestal variations. No cross-talk corrections were applied. Furthermore, the seeding and inclusion thresholds ${ }^{2}$ in the clusterisation step were set equal for all the strips on all the sensors, and no dead or noisy strip masking was applied. The C Sample data were taken in non-zero-suppressed read out mode [21]. These data were reprocessed offline with optimised parameters. The results obtained from the reprocessed data are discussed in Section 7.

\footnotetext{
${ }^{2}$ At least one strip in each cluster must have charge in ADC counts higher than the seeding threshold and all strips must have a charge higher than the inclusion threshold.
}

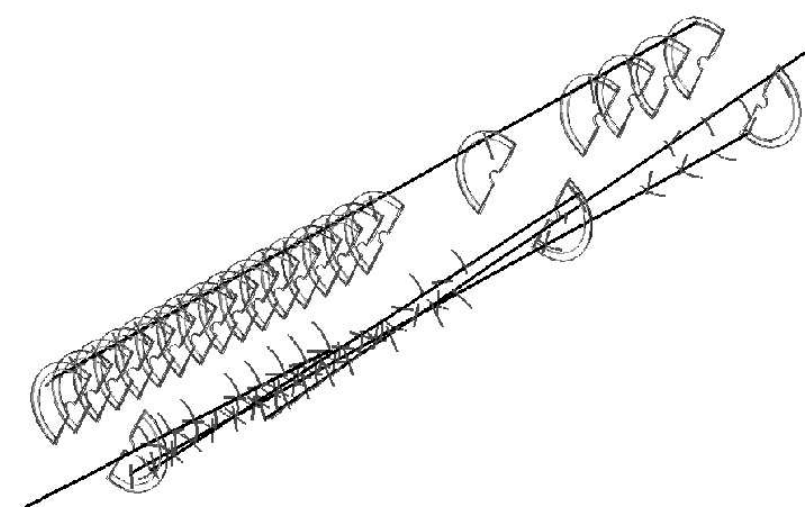

Figure 5: The event display of a typical event with reconstructed tracks in the VELO.

For the A Sample [22], on 22 August 2008, only five modules of the detector in each half were read out. The very first triggered event was observed in the VELO at 17:36 on 22 August, and tracks were reconstructed through all five planes in each half. In total around 50 events with about 60 tracks were observed during this run, which lasted approximately one hour.

Given the success of this observation, the LHC operation team agreed to provide further sets of collisions on the TED on 24 August, on 5 September and on 6 September. In these periods, all the VELO modules were powered and 76 sensors were read out. Tracks were reconstructed through up to 19 of the 21 modules in each half of the detector. A typical event is shown in fig. 5. Table 1 summarizes the data samples taken during the different synchronization tests.

\begin{tabular}{|c|c|c|c|c|}
\hline $\begin{array}{c}\text { Data Sample } \\
\text { Name }\end{array}$ & Date & $\begin{array}{c}\text { Num. Sens. } \\
\text { Read Out }\end{array}$ & $\begin{array}{c}\text { Num. of } \\
\text { Tracks }\end{array}$ & $\begin{array}{c}\text { non-zero } \\
\text { suppressed }\end{array}$ \\
\hline A Sample & $22 / 08$ & 10 & 60 & No \\
\hline B Sample & $24 / 08$ & 76 & 790 & No \\
\hline C Sample & $24 / 08$ & 76 & 60 & Yes \\
\hline D Sample & $5-6 / 09$ & 76 & 1312 & No \\
\hline
\end{tabular}

Table 1: Summary of the data taken in the VELO during the August and September 2008 TED collisions. The C Sample is a subsample of the B Sample and was the only one taken in non zero-suppressed read out mode.

\section{Timing Considerations}

The VELO requires two sets of timing parameters to be calibrated to optimally reconstruct tracks. Firstly the digitization timing must be set to correctly sample the multiplexed signal from each front-end chip. Secondly, the analogue pulse shape timing must be set up to optimise the signal sampling. The analogue signal of each channel has a rise and fall over a period of about $50 \mathrm{~ns}$, to obtain optimal timing the sampling time must be set up to within 


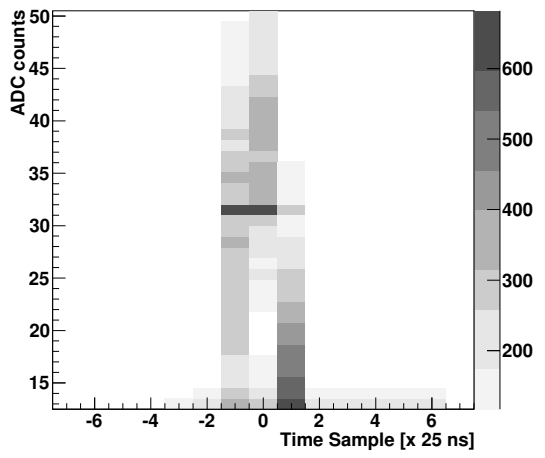

Figure 6: Total cluster charge as function of the sampling time for the B Sample. The darker colours indicate more entries.

a few ns. The timing parameters were not optimised before the TED data taking as the relative timing with respect to the beam clock can only be found using data. Nonoptimal timing significantly affects the data quality and leads to cluster finding inefficiency.

As the overall VELO timing with respect to the trigger signal could not be tested, the VELO was set up to read out several consecutive time samples for each trigger signal: 15 samples were read out for each trigger in the $\mathrm{B}$ Sample and 11 in the D Sample. The time alignment was monitored by the distribution of the total charge in ADC counts from clusters observed in all the sensors versus the time sample. Fig. 6 shows the total cluster charge in ADC counts versus the time sample for the B Sample for all $R$ sensors on the A-side. The rise and fall of the pulse shape is visible on the plot, and the timing is seen to be correct within 25 ns as the peak is observed in the central time sample.

In addition to the overall timing of the system with respect to the beam there can also be differences between modules. It is observed that some sensors have the peak of the cluster charge distribution in different time samples. The D Sample data have a faster pulse shape and lower signal compared to the B Sample data, due to different settings of the front-end chip. This gives rise to a different effect of the timing in the B and D Samples. In the analysis of the D Sample the optimal time sample was chosen for each sensor: the time sample with the highest average cluster charge was selected for each sensor. However, the timing may still be non-optimal by up to half a clock cycle (12.5 ns). Hence, no useful signal/noise results can be obtained from this analysis.

The non-optimised timing with respect to the beam and the different time calibration between sensors lead to a reduced cluster finding efficiency and degradation in resolution when taking a single central time sample for the full detector (see Section 9). Detailed automatic procedures to optimise the timing have been developed and are in place for the 2009 data taking.

\section{Track reconstruction}

Tracks were reconstructed using the two standard VELO pattern recognition algorithms $[23,24]$ run in sequence. Each event contains more tracks and clusters in the B Sample (on average 5.3 tracks and 127 clusters in the $R$ sensors) than in the D Sample (4.2 tracks and 88 clusters in the $R$-sensors). However, the D Sample taking period was longer. Overall, 790 tracks were reconstructed with at least 5 space points ${ }^{3}$ in the B Sample and 1312 tracks in the D Sample. The angular distributions of the tracks are similar in both time periods (shown in fig. 7 a) and b) for the B Sample). The distributions in both samples peak at about $10 \mathrm{mrad}$ for the angle with respect to the $z$-axis in the $x-z$ plane and at about 0 mrad for the angle with respect to the $z$-axis in the $y-z$ plane.

The reconstructed tracks cover the sensor area almost uniformly, as shown in fig. 7 c) and d). From the space point distribution along $z$ coordinate in the B Sample, the A-side has several tracks that includes space points on 13 modules and the C-side on 16 active modules. Three modules on the A-side are found to inefficiently reconstruct space points and one on the C-side: these modules have an efficiency of less than $5 \%$. The mean number of space points per track is 10.4 for the A-side ( 11.5 for the $\mathrm{C}$-side) in the B Sample and 9.3 (10.0) for the A-side (C-side) in the D Sample. The B Sample data distribution of number of space points peaks at 13 (16) for the A-side (C-side) as expected due to the track angle distribution, as shown in fig. 7. As discussed in Section 5, the non-optimised timing (even when using the best time sample for each sensor) and the high threshold give rise to loss of cluster finding efficiency in the D Sample. This is confirmed by the track pseudo-efficiency, shown for the B Sample in fig. 8. The pseudo-efficiency is defined as the ratio between the number of reconstructed points and the number of expected points. The number of expected points is obtained by extrapolating the reconstructed track to each sensor assuming all the sensors were read out and fully efficient. This pseudo-efficiency includes the hit reconstruction and the pattern recognition efficiency. The efficiency is $83 \%$ (76\%) for the C-side (A-side) in the B Sample (as shown in fig. 8 ), and $\sim 7 \%$ lower in the D Sample.

\section{Acquisition Board Algorithm Optimisation}

The data processing algorithms implemented in the processors of the TELL1 acquisition boards [11] were set to a basic configuration for these TED runs. The parameters of the algorithms were set to the same values for all strips, and not all algorithms were operated. For the final VELO operation over one million tuned parameters will be used. The configuration used for the samples in Table 1 leads to additional noise clusters in the system and

\footnotetext{
${ }^{3} \mathrm{~A} 3 \mathrm{D}$ spatial point is constructed combining radial and azimuthal hits in a module.
} 

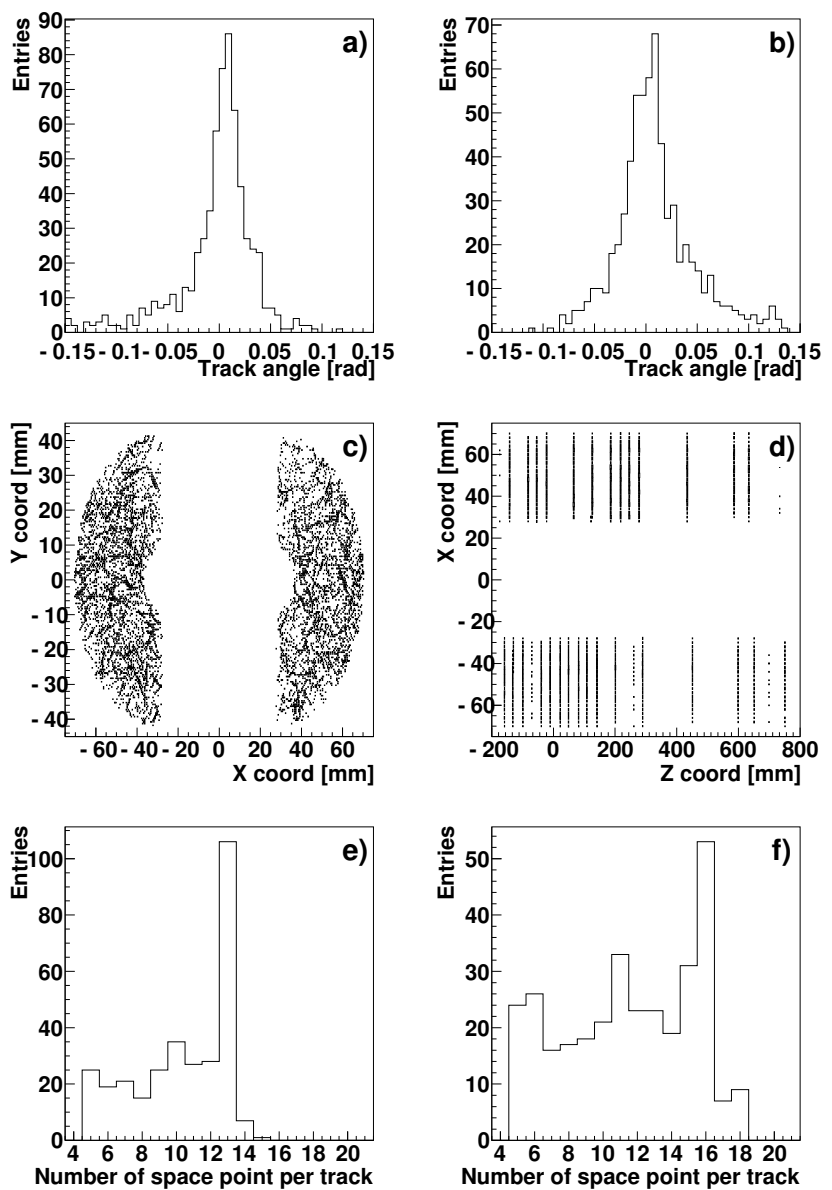

Figure 7: The angular distribution of tracks in the data samples. The a) plot shows the angle with respect to the $z$-axis in the $x-z$ plane. The b) plot shows the the angle with respect to the $z$-axis in the $y-z$ plane. The c) and d) plots show, respectively, the $x-y$ and $x-z$ coordinate of the space points associated to tracks. The e) and f) plots show the number of space points associated to a track respectively in the A-side and in the C-side. These are shown for the B Sample.

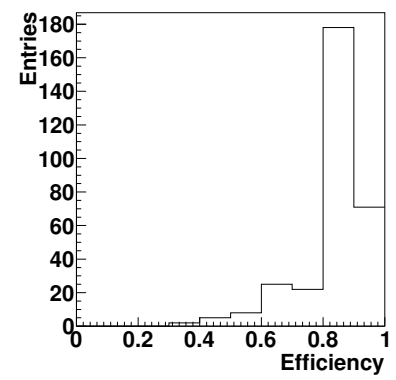

Figure 8: The track pseudo-efficiency of the reconstructed tracks evaluated as the ratio between the number of reconstructed space-points and the number of expected ones. This is shown for the C-side for the B Sample. a loss of efficiency for finding signal clusters. (This is in addition to the loss of cluster finding efficiency caused by the non-optimised timing discussed in Section 5.) Indeed, one might naively expect that the data quality would be very poor as a result of using the same parameters for all strips in the pedestal algorithm rather than subtracting the correct value for each strip. Hence, this could lead to clusters being produced from the ADC difference between the true pedestal and the subtracted value. However, this effect is ameliorated by the use of the common mode correction algorithms that partially remove the variation of the pedestals.

A small fraction $(\sim 3 \%)$ of the data was taken without zero suppression for the full detector in addition to the standard zero suppressed data. This non-zero suppressed data sample has been used to evaluate the consequences of non-tuned FPGA algorithms. The non-zero suppressed data were processed offline with the Vetra [21] data reconstruction software which emulates the performance of the TELL1 readout board processing algorithms to obtain the cluster data collection. The emulation was run with the appropriate pedestal values, Beetle header cross-talk correction parameters and clusterisation thresholds. Individual pedestal values for each channel were obtained using noise data with random triggers around the time of the TED data runs, using the procedure described in [25]. These values were then subtracted in the reprocessing of the non-zero suppressed data. Beetle header cross-talk correction parameters were calculated with the same data sample using the procedure described in [26]. Two common mode correction algorithms were applied: mean common mode subtraction (applied before the reordering [21]) and linear common mode subtraction (performed after the reordering). The cluster seeding, inclusion and spillover thresholds were evaluated for each individual strip using the noise data run. The seeding threshold was set to 3.5 times the measured noise. The inclusion thresholds were set to $40 \%$ of the seeding threshold in each channel. The spillover thresholds (one per analogue link) were fixed and equal to $20 \mathrm{ADC}$ counts.

The reprocessed sample, including 70 tracks, was compared with the original TELL1 cluster output. The total cluster charge for both data samples are shown in fig. 9 . The quality of the cluster charge distributions are significantly improved after reprocessing, improving the Landau distribution by removing the isolated peaks. The peak at low cluster charges is due to the noise. With the tuned processing parameters the distribution of number of strips in the cluster approximately agrees with the simulation.

The number of clusters on the track as a function of the sensor number ${ }^{4}$ is shown in fig. 10. After reprocessing the number of space points associated to a track is found to increase by about $16 \%$, and about $17 \%$ more tracks are found. The cluster finding efficiency, evaluated as the ratio

\footnotetext{
${ }^{4}$ The sensor number increases along $z$.
} 

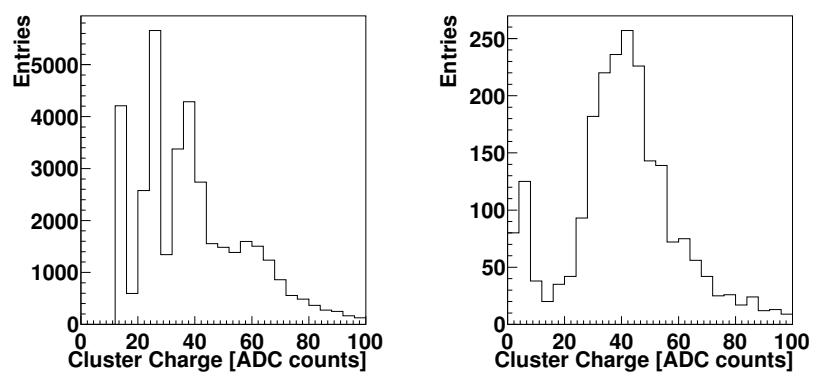

Figure 9: The cluster charge in the $\Phi$ sensors for the C Sample. The left hand histogram is for the clusters produced by the TELL1 processing. The right histogram shows the clusters obtained by reprocessing the non-zero suppressed data offline to obtain clusters using a tuned emulation of the algorithms.
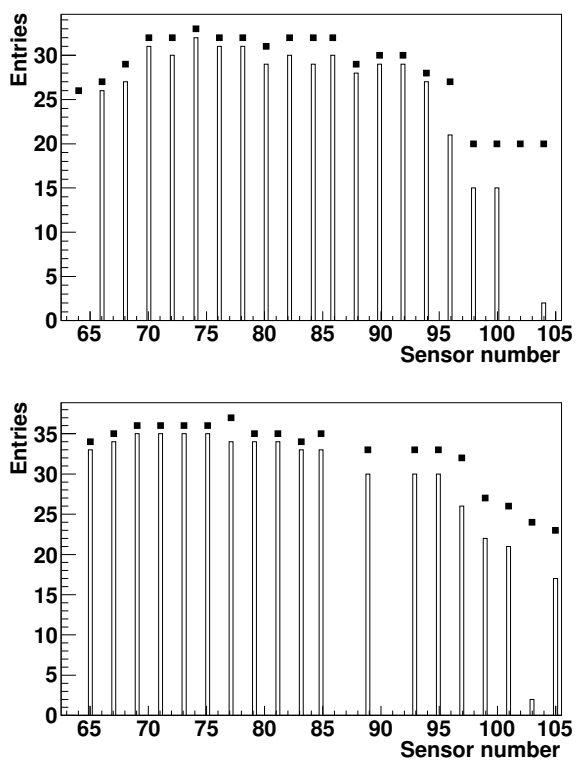

Figure 10: The number of $\phi$ clusters associated to tracks versus the sensor number for the zero-suppressed-data (histogram) and the reprocessed non-zero suppressed data (black boxes), for the $\mathrm{C}$ Sample. The top plot is for the sensors on the A-side and the bottom plots for the sensors on the $\mathrm{C}$-side.

between the number of measured hits and the expected number of points per track for fully efficient sensors, only increases by about $1.5 \%$ overall. However, the reprocessing markedly improves the cluster finding efficiency for some sensors. The maximum number of points associated to a track increases up to 19 for tracks reconstructed in the C-side.

In summary, the basic configuration of the TELL1 algorithms used for the TED run leads to a loss in cluster finding efficiency and an increase in noise clusters in the system. The data quality can be improved by tuning the algorithms, as demonstrated with the reprocessing of the non-zero suppressed data. Calibrated algorithms will be utilized during data taking with colliding beams.

\section{Spatial Alignment}

The alignment of the VELO relies on three components: the precision construction and assembly of the detector; the survey of the individual modules and the assembled system; and the software alignment of the system using tracks. The sensor positions were measured to an accuracy better than $10 \mu \mathrm{m}[27]$ and they provide the starting position for the VELO software alignment.

The software alignment procedure for the detector is divided in three steps [28]: 1) alignment of the relative position of the $R$ and $\Phi$ sensors; 2 ) alignment of the modules within each of the VELO-halves; 3 ) relative alignment of the two halves with respect to each other.

The first stage, the relative alignment of the two sensors in each module [29], is performed by a fit to the shape of the residual distribution as a function of the azimuthal angle and the radius for each sensor. This method requires an order of magnitude more statistics than that collected in the data discussed here.

The second stage, the module alignment in each half [30], is performed by a non-iterative method using matrix inversion handled by the Millepede program [31]. The alignment is based on a $\chi^{2}$ function produced from the residuals between the tracks and the measured clusters. The number of tracks required for an effective alignment is relatively modest, and an initial alignment can be obtained even using this small collected data sample (the B or D Sample).

The final stage, the relative alignment of the two halves [32], is constrained by tracks traversing both halves and by the reconstruction of primary vertices. No such tracks are available in this data sample.

Hence, this Section reports the first alignment of the relative module positions in the VELO, and compares this with survey data. Previous results on the alignment of the partially assembled system based on test beam data are reported in [13].

The module alignment constants were determined using the B and D Sample selecting tracks having $\chi^{2} / D O F$ smaller than 100 and with at least 5 space points per track, after rejecting the hit outliers by a biased residual cut at $300 \mu \mathrm{m}$ for the $R$-sensor and $60 \mathrm{mrad}$ for the azimuthal sensors. The alignment constants were determined for 29 of the 42 VELO modules. This corresponds to the number of modules where both $R$ and $\Phi$ sensors were read out, and excludes four modules that have low cluster finding efficiency (less than 5\%) in one of the sensors (see Section 7). Only the degrees of freedom to which the relative module alignment is primarily sensitive are considered: the translations of the modules in the $x$ and $y$ directions; and the rotation around the $z$-axis.

A test of the convergence of the Millepede method was performed applying the procedure for 5 consecutive iterations. In each iteration the full event reconstruction is performed, including the pattern recognition. The average absolute change in alignment constants between itera- 

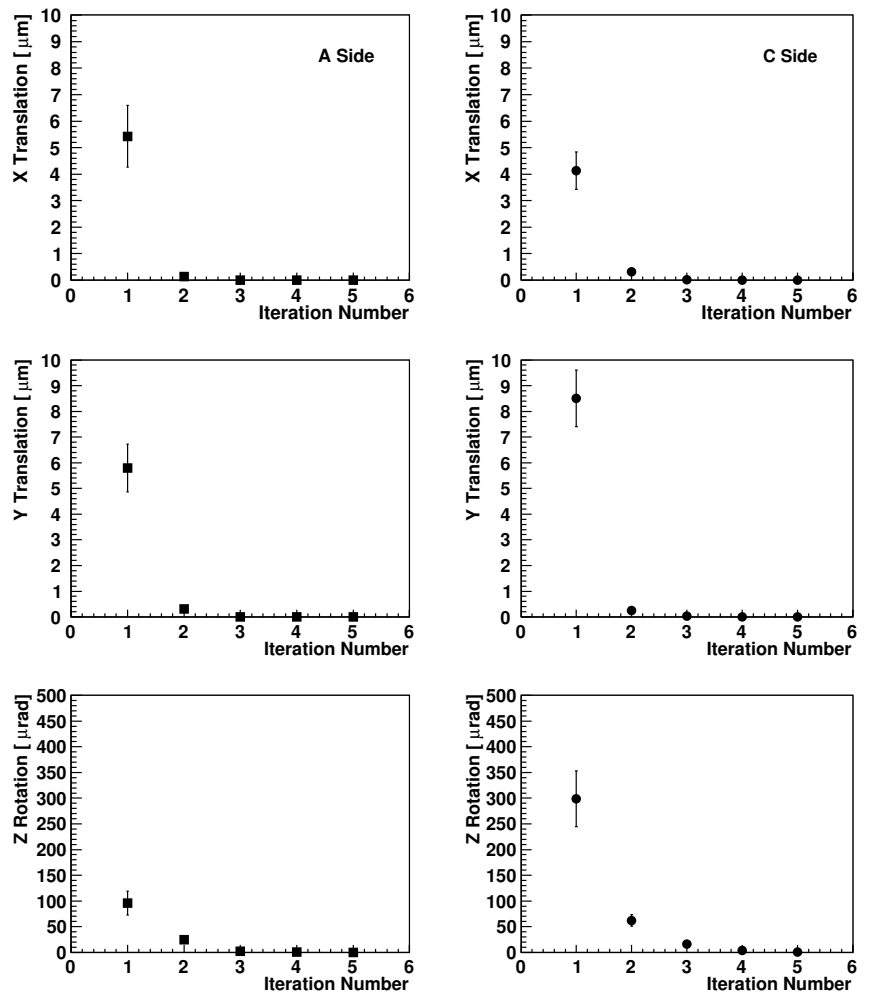

Figure 11: The average absolute change in alignment constants between iterations of the alignment method is shown versus the iteration number. The two columns show the results for the A-side (left) and the $\mathrm{C}$-side (right). The three rows show the different alignment constants: $x$ translations are in the first row; $y$ translations in the second row; and rotations around the $z$-axis in the third row.

tions is plotted versus the iteration number in fig. 11 . The change shown for the first iteration, is with respect to the survey alignment constants. The method converges after the first iteration for all 3 degrees of freedom. The alignment procedure, for a single iteration, takes around 0.2 seconds on a single $5 \mathrm{GHz}$ CPU.

The alignment constants were evaluated separately for the B and D Sample. The average absolute difference between the alignment constants obtained and the survey values is about $5 \mu \mathrm{m}$, with a maximum of $20 \mu \mathrm{m}$, for translations along $x$ and $y$ coordinates. For rotations around the $z$-axis the difference is about $200 \mu \mathrm{rad}$, with a maximum of 600 urad. This is shown in fig. 12 .

The results obtained from the B and D Sample are in good agreement for $x$ and $y$ translations. From the distribution of the difference of the alignment constants between the B and D Sample (shown in fig. 13), the accuracy of the alignment was estimated. A precision of $2.7 \mu \mathrm{m}$ for translations along $x$ and $y$ coordinates and $178 \mu \mathrm{rad}$ for rotations around the $z$-axis was obtained. The larger difference in alignment constants for the rotation around the $z$-axis is probably due to the high noise rate in $\Phi$ sensors in the B Sample. This alignment parameter is determined
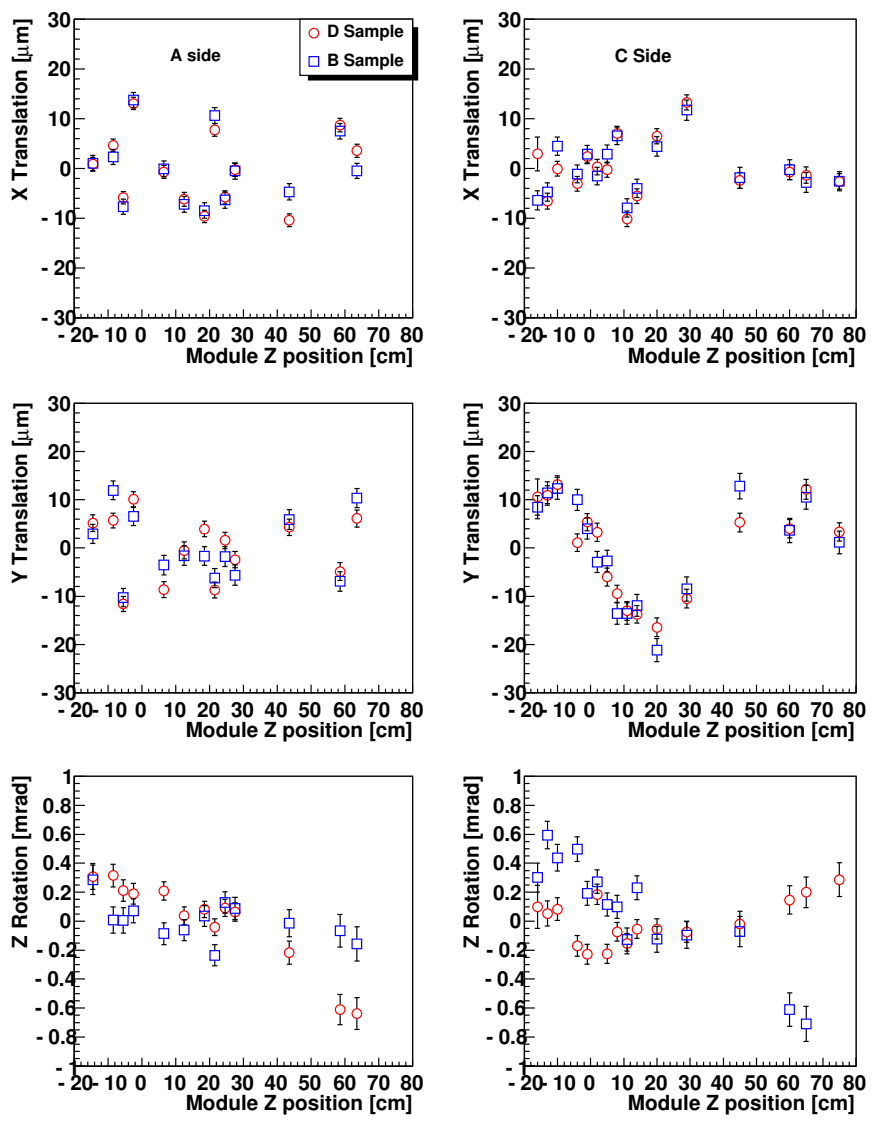

Figure 12: The difference between the alignment constants obtained from the data by the Millepede method and the survey measurements. The B Sample (squares) and the D Sample (circles) results are both shown. The two columns show the results for the A-side (left) and the C-side (right). The three rows show the different alignment constants: $x$ translations are in the first row; $y$ translations in the second row; and rotations around the $z$-axis in the third row. 

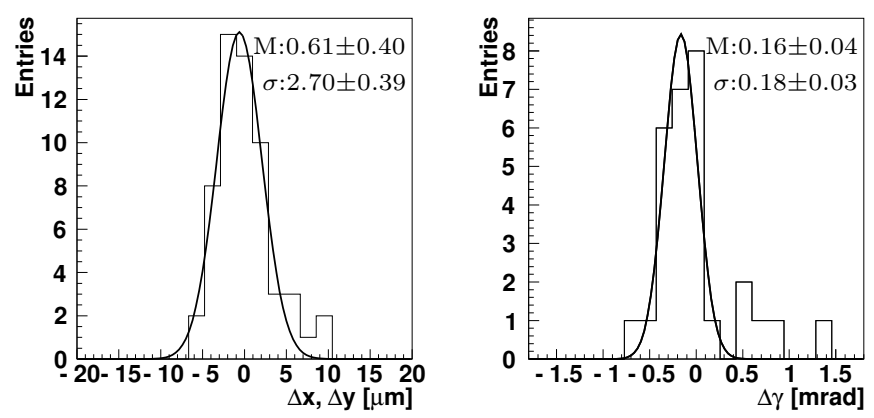

Figure 13: The difference between the alignment constants evaluated by the Millepede method using the B Sample and the D Sample. The left hand plot shows the difference of alignment constants for the $x$ and $y$ translations, the right hand plot is for the rotation around the $z$-axis.

by the $\phi$ residuals. The alignment parameters were also determined using only $x$ and $y$ translations. The results obtained are in good agreement, within errors, with those obtained including the rotation around the $z$-axis. Hence, the misalignment of the rotation with respect to the survey is confirmed to be small.

The results obtained using the method based on Millepede, were also compared with those obtained using an alternative method. The method based on Millepede uses a standalone straight line track fit, while the alternative method uses the default LHCb tracks fit based on a Kalman filter [33]. The results obtained from the two methods are compatible and in good agreement within the current alignment precision, as shown for the D Sample in fig. 14 .

In order to check whether the alignment constants are being biased by the starting values, the alignment was run starting from the nominal position of the modules (rather than their survey measurement positions). The module alignment by tracks reconstructed in a single VELO half cannot determine any translations or rotations due to a global translation, rotation, shearing or twist of the system. To subtract this global effect we fit the difference of alignment constants by a straight line (corresponding to a global translation or rotation) and evaluate the difference to this line. The difference after subtracting the global translation rotation effect is shown in fig. 15. The reproducibility of the alignment from different starting conditions is seen to be within $5 \mu m$ for $x$ and $y$ translations and $200 \mu \mathrm{rad}$ for $z$ rotations.

The improvement of the data quality using the new alignment constants, rather than taking the survey constants, has been evaluated. A small improvement has been observed. The average number of points per track increases by about $1 \%$. The average $\chi^{2}$ of a track (shown in fig. 16) improves by about $3 \%$. The RMS of the pull distribution for $r$ and $\phi$ residuals decreases by $5 \%$. The differences obtained using the alignment constant sets from
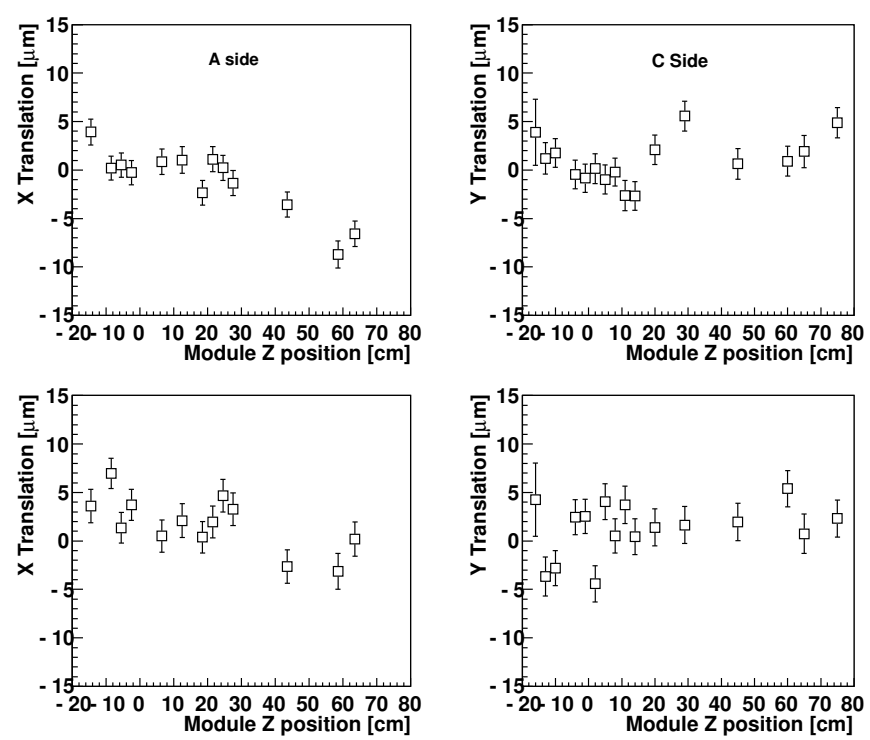

Figure 14: The difference between the alignment constants evaluated using the D Sample by a method based on Millepede and a method based on a Kalman fit. The two columns show the results for the A-side (left) and the $\mathrm{C}$-side (right). The difference of $x$ translations are in the first row; and the difference of $y$ translations are in the second row.

B or D Sample are within the statistical error. Similarly the alignment results obtained using the method based on Millepede and the ones based on the Kalman filter are of equal quality on these data sample.

In summary, the software alignment confirms that the modules have not been significantly displaced from their survey positions during transport, insertion, evacuation, cooling down or subsequently in the system. The module alignment precision obtained from this data sample is about $5 \mu \mathrm{m}$ for $x$ and $y$ translation and $200 \mu \mathrm{rad}$ for rotations around the $z$-axis.

\section{Spatial Resolution}

The VELO sensor resolution has been determined using the B Sample. The hit residuals are evaluated using a straight line track fit; both a simple straight line fit and a Kalman filter track fit (without any correction for multiple scattering and energy losses) have been used and shown to agree. The residual is defined by the distance between the hit measurement and the extrapolated point to that sensor of the fitted track including the hit measurement. Using the point for which the residual is being determined in the track fit gives rise to a bias in the residual. This has been corrected to evaluate the hit resolution. This correction is derived to be $\sqrt{V / R}$ [33] where $\mathrm{V}$ is the variance of measurement and $R$ is the variance of residual. The evaluation of this correction is implemented in the Kalman fit. This has been cross-checked by explicitly excluding the sensor from the fit to obtain an unbiased residual and correcting it for the error due to the extrapolation of the track to 

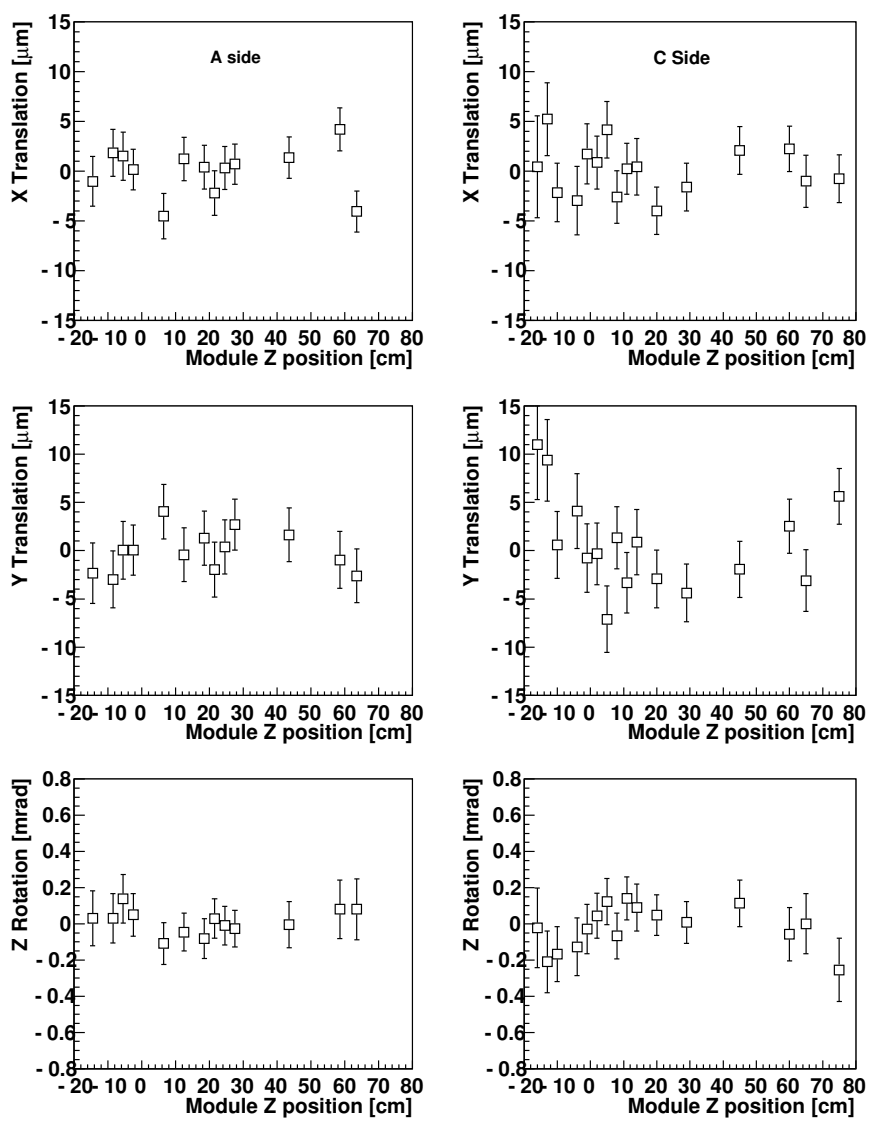

Figure 15: The difference between the alignment constants evaluated with the Millepede method for the D Sample using two different initialization module constants. The initialization values for the Millepede algorithm were taken from the survey or from the nominal positions. The effect of global translations or rotations of each half has been subtracted. The two columns show the results for the A-side (left) and the C-side (right). The three rows show the different alignment constants: $x$ translations are in the first row; $y$ translations in the second row; and rotations around the $z$-axis in the third row.

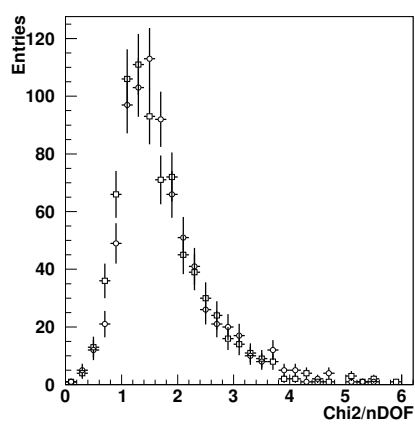

Figure 16: The $\chi^{2} / D O F$ distribution before (circles) and after (squares) track-based alignment for the B Sample.

the sensor [34] . The resolution has been determined from the sigma of a Gaussian fit to the distribution of the corrected residuals. The resolution is extracted as a function of the local strip pitch at the track intercept point. The resolution is evaluated using tracks with at least 16 hits (corresponding to 8 space points).

Moreover, tracks with a kink due to multiple scattering inside the VELO are excluded by cutting on the track angle. Only the tracks in the central peak of the angular distribution are selected: the tracks are required to have angles in the $x-z$ plane between $-15 \mathrm{mrad}$ and $20 \mathrm{mrad}$ and angles in the $y-z$ plane between $-20 \mathrm{mrad}$ and 10 mrad.

Fig. 17 shows the VELO sensor resolution, averaged over all $R$ and $\Phi$ sensors. A single hit precision of roughly $12 \mu \mathrm{m}$ for a pitch of $40 \mu \mathrm{m}$ is obtained for both $R$ and $\Phi$ sensors. The resolution is equivalent to binary resolution, which is consistent with the fraction of single strip clusters of $85 \%-90 \%$ observed in this data sample. With the properly tuned TELL1 parameters and the correct time alignment, the resolution obtained for tracks with such an angular distribution is expected to be equal or better than that obtained from the test beam measurements [13].

A significantly better resolution [12] is expected for tracks at angles around $140 \mathrm{mrad}$, for which the charge sharing between adjacent strips is optimal. The resolution has been extracted using a simple weighted pulse height algorithm for reconstruction of the cluster position. Additional development of the clustering algorithm is expected to further improve the precision.

\section{Conclusion}

The first LHC beam induced tracks have been reconstructed in the LHCb VELO. The reconstruction, tracking and alignment algorithms have been successfully tested with TED data and the VELO detector performance assessed. This data sample has significantly aided the commissioning of the LHCb VELO. 

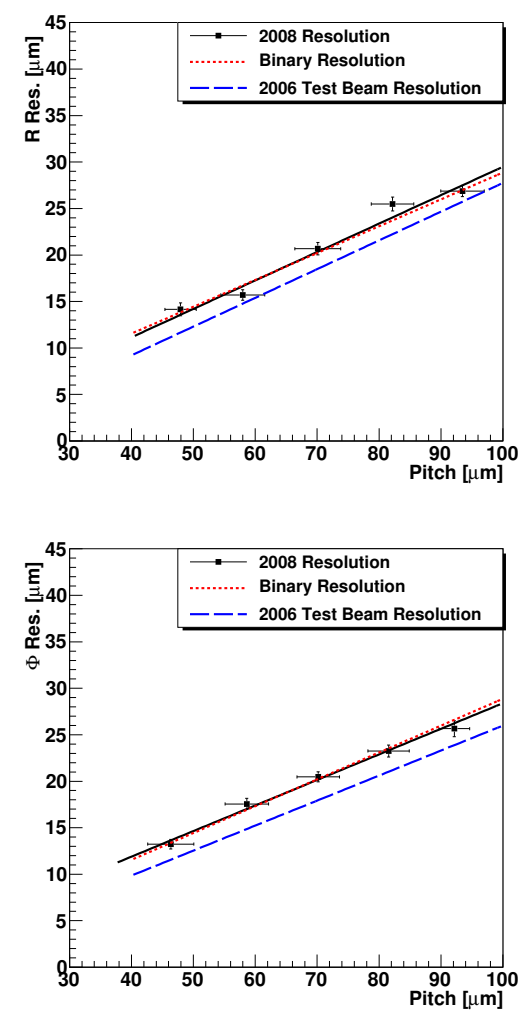

Figure 17: The VELO resolution for the $R$ (top plot) and $\Phi$ (bottom plot) sensors obtained with the B Sample as a function of the readout pitch, compared with binary resolution and previous test beam measurements [13].
Approximately 2200 tracks have been reconstructed from particles produced in the interaction of a single proton bunch on a beam absorber. The data were taken during synchronisation tests of the LHC in August and September 2008. The tracks have a small angle and traverse a large number of VELO sensors. This sample has allowed to study the VELO detector performance.

The data quality is found to be affected by the nonoptimised timing set-up and data processing algorithm tuning. These lead to reduced cluster finding efficiencies and increased numbers of noise clusters. The timing and processing parameters of the final VELO system will be optimised with the first data to improve the VELO reconstruction quality. The small data sample available with full raw data has been reprocessed with a more optimised reconstruction algorithm tuning. The reprocessed raw data is shown to have a higher hit efficiency and lower noise than in the zero suppressed data.

The relative module alignment has been determined using these data. The modules are found to be displaced from their surveyed positions by less than $10 \mu \mathrm{m}$. The precision of the software alignment is about $5 \mu \mathrm{m}$ for $x$ and $y$ translation and $200 \mu \mathrm{rad}$ for the rotation around the $z$-axis.

The measured position resolution is compatible with binary expectation, which is consistent with the majority of clusters being composed of a single strip. Improvements are expected with the correctly tuned TELL1 parameters and the optimised time alignment.

\section{Acknowledgments}

We gratefully acknowledge the assistance of all our colleagues on the LHCb experiment. This successful observation critically relied on the triggering of the experiment from the calorimeter and the online data acquisition system. We are grateful to Wouter Hulsbergen for providing the alignment results obtained using the Kalman filter method. We also wish to thank the LHC operations team for their assistance in preparing these tests, and keeping us updated with beam conditions.

\section{References}

[1] The ALICE Collaboration, K. Aamodt et al., J. Instrum. 3 (2008) S08002

[2] The ATLAS Collaboration, J. Instrum. 3 (2008) S08003

[3] The CMS Collaboration, J. Instrum. 3 (2008) S08004

[4] The LHCb Collaboration, CERN/LHCC 2003-030 (2003)

[5] C. Lippmann, for the ALICE Collaboration, arXiv:0905.1565 (2009)

[6] M. Ishino for the ATLAS Collaboration, ATL-GEN-PROC2009-006 (2009)

[7] I. Mikulec for the CMS Collaboration, CMS-CR-2009-026 (2009)

[8] S.Blusk et al., CERN-2007-004 (2007)

[9] The LHCb Collaboration, CERN/LHCC 2001-011 (2001)

[10] The LHCb Collaboration, J. Instrum. 3 (2008) S08005

[11] G. Haefeli et al., Nucl. Instrum. and Meth. A 560 (2006) 494

[12] T. Szumlak, C. Parkes, T. Ruf, LHCb-2007-151 
[13] M. Gersabeck et al., Nucl. Instr. and Meth. A596 (2008) 164

[14] L. Ducimetire et al., Proc. IEEE Pulsed Power Conference, Albuquerque, USA, July 10-13, 1995

[15] S.Praire and P.R. Sala LHC Project Report 465 (2001)

[16] H. Vincke and G. R. Stevenson, CERN-TIS-2003-013-RP-TN (2003)

[17] M. Benedikt et al., CERN 2004-003 (2004)

[18] O.Bruening et al., LHC Project Report 291 (1999)

[19] M. Agari et al., Nucl. Instr. Meth. A 518 (2004) 468

[20] G. Haefeli et al., LHCb-2003-007

[21] T. Szumlak, C. Parkes, LHCb-2008-022

[22] C. Parkes, S. Borghi et al., Nucl. Instr. Meth. A 604 (2009) 1

[23] D. Hutchcroft, LHCb-2007-013

[24] T. Lastovicka, LHCb-2007-002

[25] C. Parkes, T. Szumlak, LHCb-2009-036

[26] T. Szumlak, C. Parkes, LHCb-Note in preparation

[27] M. Gersabeck, LHCb-2008-044

[28] S. Viret, C. Parkes, M. Gersabeck, Nucl. Instr. and Meth. A596 (2008) 157

[29] M. Gersabeck, C. Parkes, S. Viret, LHCb-2007-138

[30] S. Viret, C. Parkes, D. Petrie, LHCb-2005-101

[31] V. Blobel and C. Kleinwort, Contribution to the Conference on Advanced Statistical Techniques in Particle Physics PHYSTAT2002, Durham U.K., hep-ex/0208021

[32] S. Viret, C. Parkes, M. Gersabeck, LHCb-2007-067

[33] W. Hulsbergen, Nucl. Instr. Meth. A 600 (2009) 471

[34] P. Bartalini et al., LHCb-2000-099 